\title{
Asymptotically Exact Approximations for the Symmetric Difference of Generalized Marcum $Q$-Functions
}

\author{
F. Javier Lopez-Martinez, Member, IEEE, and Juan M. Romero-Jerez, Senior Member, IEEE
}

\begin{abstract}
In this paper, we derive two simple and asymptotically exact approximations for the function defined as $\Delta \mathcal{Q}_{m}(a, b) \triangleq Q_{m}(a, b)-Q_{m}(b, a)$. The generalized Marcum $Q$ function $Q_{m}(a, b)$ appears in many scenarios in communications in this particular form, referred to as symmetric difference of generalized Marcum Q-functions or difference of generalized Marcum $Q$-functions with reversed arguments. We show that the symmetric difference of Marcum $Q$-functions can be expressed in terms of a single Gaussian $Q$-function for large and even moderate values of the arguments $a$ and $b$. A second approximation for $\Delta \mathcal{Q}_{m}(a, b)$ is also given in terms of the exponential function. We illustrate the applicability of these new approximations in different scenarios: (a) Statistical characterization of Hoyt fading, (b) Performance analysis of communication systems, (c) Level crossing statistics of a sampled Rayleigh envelope, and (d) Asymptotic approximation of the Rice $I_{e}$ function.
\end{abstract}

Index Terms-Asymptotic analysis, Cumulative distribution function, Diversity reception, Gaussian $Q$-function, Level crossing rate, Marcum $Q$-function, Rice $I_{e}$-function.

\section{INTRODUCTION}

The generalized Marcum $Q$-function ${ }^{1}$ is extensively used in the context of communication theory since its inception more than 60 years ago. This integral function is defined in [1, 2] as an incomplete integral involving powers, exponentials and modified Bessel functions of the first kind according to the following expression

$$
Q_{m}(a, b)=\frac{1}{a^{m-1}} \int_{b}^{\infty} x^{m} e^{-\frac{x^{2}+a^{2}}{2}} I_{m-1}(a x),
$$

and it was first used to characterize the probability of target detection by pulsed radar [3]. This function also appears in some of the most relevant scenarios in communication theory: the cdf of the non-central chi-squared distribution, the Rayleigh bivariate cdf [4], the Nakagami- $m$ bivariate cdf $[5,6]$, or in the characterization of quadratic forms in complex

Copyright (C)2014 IEEE. Personal use of this material is permitted. However, permission to use this material for any other purposes must be obtained from the IEEE by sending a request to pubs-permissions@ieee.org.

The work of F. J. Lopez-Martinez was supported by Junta de Andalucia (P11-TIC-7109), Spanish Government (TEC2010-18451, COFUND201340259), Univ. Malaga and EU under Marie-Curie COFUND U-mobility program (ref. 246550). The work of J. M. Romero-Jerez was supported by European FEDER funds and the Spanish Ministry of Economy and Competitiveness, grants TEC2009-13763-C02-01 and TEC2013-42711-R.

F. J. Lopez-Martinez is with Dept. of Electrical Engineering, Stanford University, Stanford CA, 940305 (email: fjlm@stanford.edu).

J. M. Romero-Jerez is with Dpto. Tecnología Electrónica, Universidad de Malaga, Spain (email: romero@dte.uma.es).

${ }^{1}$ In the literature, the term Marcum $Q$-function is used to denote the special case $m=1$, whereas the generalized Marcum refers to this function with arbitrary value of $m$. For the sake of readability, we will use Marcum $Q$-function to denote $Q_{m}(\cdot)$; a distinction will be specifically made when necessary for clarification purposes.
Gaussian random variables [7] just to name a few. Ever since its appearance, the Marcum $Q$-function has been a matter of study by many authors in the literature due to its paramount importance. This function is typically a built-in function in commercial mathematical software packages. However, it does not allow to gain insight on system performance in a simple way due to its complicated nature. For this reason, many authors have focused on the derivation of simple lower and upper bounds for this function [8-12].

In some scenarios, the Marcum $Q$-function appears in the following particular form

$$
\Delta \mathcal{Q}_{m}(a, b) \triangleq Q_{m}(a, b)-Q_{m}(b, a) .
$$

This form, referred to as difference of Marcum $Q$-functions with reversed arguments [13, 14] or symmetric difference of Marcum Q-functions [15], appears for instance when characterizing the bit error rate (BER) performance of digital communication systems with quadratic-form receivers [7, 13]. On a different context, the cdf of the Nakagami- $q$ or Hoyt distribution [15] or the Rice $I_{e}$ function [16] are also related with the symmetric difference of Marcum $Q$-functions.

The specific nature of the function $\Delta \mathcal{Q}_{m}(a, b)$ affects the validity and range of application of some of the existing bounds and approximations known for the Marcum $Q$ functions. This aspect was addressed by Simon and Alouini in $[8,14]$, stating that the combination of lower and upper bounds for the individual $Q_{m}$ functions to upper-bound the difference of Marcum $Q$-functions with reversed arguments would yield to a looser upper bound in most circumstances (e.g. including Chernoff bounds). A similar conclusion can be extracted when studying the asymptotic approximations for the Marcum $Q$ function [17]; when the accuracy of the approximation relies on the relative magnitude of the parameters $a$ and $b$, the approximation is degraded as the second argument in one of the Marcum $Q$-functions is necessarily lower than the first argument. Despite the importance of the symmetric difference of Marcum $Q$-functions in communication theory, its asymptotic properties have not been tackled in the bibliography to the best of our knowledge.

In this paper, we derive a simple, elegant yet very accurate approximation for the symmetric difference of generalized Marcum $Q$-functions. The proposed expression is given in terms of a single Gaussian $Q$-function scaled by a weighting factor, and is asymptotically exact for large values of the arguments $a$ and $b$. We also propose a second approximation in terms of the exponential function, making use of the asymptotic properties of the Gaussian $Q$-function. Our approximations allow to obtain interesting insights in many scenarios of interest in communication theory: 
- When applied to approximate the Nakagami-q (Hoyt) distribution function [15], we obtain an asymptotically exact approximation for the cdf in terms of the Gaussian $Q$-function for low values of $q$. Since the Hoyt distribution reduces to the one-sided Gaussian for $q=0$, our approximation shows how the Hoyt cdf tends to have the form of a Gaussian $Q$-function as $q \rightarrow 0$, which is intuitively meaningful.

- We find a simple asymptotic approximation for the BER performance of multichannel reception of differentially coherent and non-coherent detection of phase-shift keying and frequency-shift keying signals [13]. Using a further asymptotic relationship between the Gaussian $Q$-function and the exponential function, we can easily appreciate an exponential decay in the BER as the signal-to-noise (SNR) tends to infinity.

- We find a simple approximation for the level crossing rate (LCR) of a Rayleigh fading envelope sampled at period $T_{s}$ [18]. We observe how the proposed approximation gives very accurate results for large values of the threshold level, and reduces to the well-known Rice's formula [19] as $T_{s} \rightarrow \infty$.

- The Rice $I_{e}$ function admits a representation in terms of the symmetric difference of Marcum $Q$-functions [16]. Hence, we provide two simple approximated expressions for this function.

\section{ASYMPtOTIC ANALYSIS}

\section{A. Notation and preliminaries}

In the following, we use $|\cdot|$ to indicate the modulus of a complex number. The cdf of a random variable $X$ is defined as $F_{X}(x) \triangleq \operatorname{Pr}\{X \leq x\}$, and consequently, the complementary cdf (ccdf) of $X$ is defined as $\bar{F}_{X}(x) \triangleq 1-F_{X}(x)$. The joint cdf of two correlated random variables $X$ and $Y$ is defined as $F_{X, Y}(x, y) \triangleq \operatorname{Pr}\{X \leq x, Y \leq y\}$.

\section{B. Results}

Lemma 1: Let $\Delta \mathcal{Q}_{m}(a, b)$ be the symmetric difference of two generalized Marcum $Q$-functions as defined in (2). Then, the function $\Delta \mathcal{Q}_{m}(a, b)$ can be approximated for large values of its arguments $a$ and $b$ by

$$
\Delta \mathcal{Q}_{m}(a, b) \approx 1-\kappa_{m}(a, b) Q(a-b) \triangleq \overline{\Delta \mathcal{Q}}_{m}(a, b),
$$

where $Q(\cdot)$ is the Gaussian $Q$-function and the coefficient $\kappa_{m}(a, b)$ is expressed as

$$
\kappa_{m}(a, b)=\left(\frac{a}{b}\right)^{m-0.5}+\left(\frac{a}{b}\right)^{0.5-m},
$$

and $a>b \geq 0, m \in \mathbb{N}$.

Proof: See Appendix A.

Note that $a>b$ does not actually pose any restriction on the approximation when $b>a$. In this case, since $\Delta \mathcal{Q}_{m}(a, b)=-\Delta \mathcal{Q}_{m}(b, a)$, we can approximate $\left.\Delta \mathcal{Q}_{m}(a, b)\right|_{b>a} \approx-\left.\overline{\Delta \mathcal{Q}}_{m}(b, a)\right|_{b>a}$. This asymptotic approximation is not explicitly reported in the literature to the best of our knowledge and is valid for a positive integer value of $m$, which is the usual case in communication theory. It is interesting to note that the argument of the Gaussian $Q$ function is independent of $m$. Two direct implications arising from this lemma are presented in the following corollaries.

Corollary 1: The symmetric difference of two first-order Marcum $Q$-functions can be approximated for large values of its arguments $a$ and $b$ by

$$
\Delta \mathcal{Q}_{1}(a, b) \approx 1-\left\{\sqrt{\frac{a}{b}}+\sqrt{\frac{b}{a}}\right\} Q(a-b) .
$$

Proof: Substituting $m=1$ in (3) directly yields (5).

Corollary 2: The symmetric difference of two generalized Marcum $Q$-functions can be approximated for large values of its arguments $a$ and $b$ by

$$
\Delta \mathcal{Q}_{m}(a, b) \approx 1-\kappa_{m}(a, b) \frac{e^{-\frac{(a-b)^{2}}{2}}}{\sqrt{2 \pi}(a-b)} .
$$

Proof: Using the well-know relation with the complementary error function $Q(z)=\frac{1}{2} \operatorname{erfc}(z / \sqrt{2})$, and the first term of the asymptotic expansion of the complementary error function $\operatorname{erfc}(z)=\frac{e^{-z^{2}}}{\sqrt{\pi} z}+\mathcal{O}\left(z^{-3} e^{-z^{2}}\right), z \rightarrow \infty$ in [20, eq. 7.1.23] we directly obtain (6).

\section{APPROXIMATION ERROR}

After presenting in the previous section the approximations for the symmetric difference of generalized Marcum $Q$ functions, we evaluate their range of validity in terms of the values of $a, b$ and $m$. For convenience of discussion ${ }^{2}$ we will adopt the notation used in [8], where the parameters $a=\alpha x$ and $b=x$, where $\alpha>1$. We define the relative approximation error as

$$
\varepsilon_{m}(\alpha, x) \triangleq\left|\frac{\Delta \mathcal{Q}_{m}(\alpha x, x)-\overline{\Delta \mathcal{Q}}_{m}(\alpha x, x)}{\Delta \mathcal{Q}_{m}(\alpha x, x)}\right| .
$$

In the derivation process detailed in the Appendix, it is assumed that the argument $x$ is sufficiently large in order to approximate the Marcum $Q$-function as a scaled Gaussian $Q$ function. It is also assumed that the product $\alpha x^{2}$ is also sufficiently large as to approximate the modified Bessel function of the first kind as a scaled exponential function. Hence, we will study the approximation error given by $\varepsilon_{m}(\alpha, x)$ in terms of the parameters $\alpha$ and $x$. In Fig. 1, we represent $\varepsilon_{1}(\alpha, x)$ for different values of $\alpha$, considering the approximations introduced in (3) and (6). When the Gaussian $Q$-function approximation is used, for small values of $\alpha$, we observe how a relatively large value of $x \approx 3.5$ is required to obtain an error in the range of $1 \%$. It is noted how for values of $\alpha>1.5$ the approximation error is very low even for not-solarge values of $x$. In the case of the exponential approximation, the approximation error for small $\alpha$ is very large; in fact, the curve corresponding to $\alpha=1.01$ is out of the plot. However, for reasonably large values of $\alpha$ this approximation behaves similarly to the one given by (3).

\footnotetext{
${ }^{2}$ To the best of our knowledge, in all the scenarios where the symmetric difference of Marcum $Q$-functions makes appearances, it has the form $\Delta \mathcal{Q}_{m}(\xi u, \beta u)$ or $\Delta \mathcal{Q}_{m}(\xi \sqrt{\gamma}, \beta \sqrt{\gamma})$, with $\xi>\beta$. This is observed in the scenarios addressed in Section IV, where $\xi$ and $\beta$ remain constant once the system parameters have been fixed.
} 


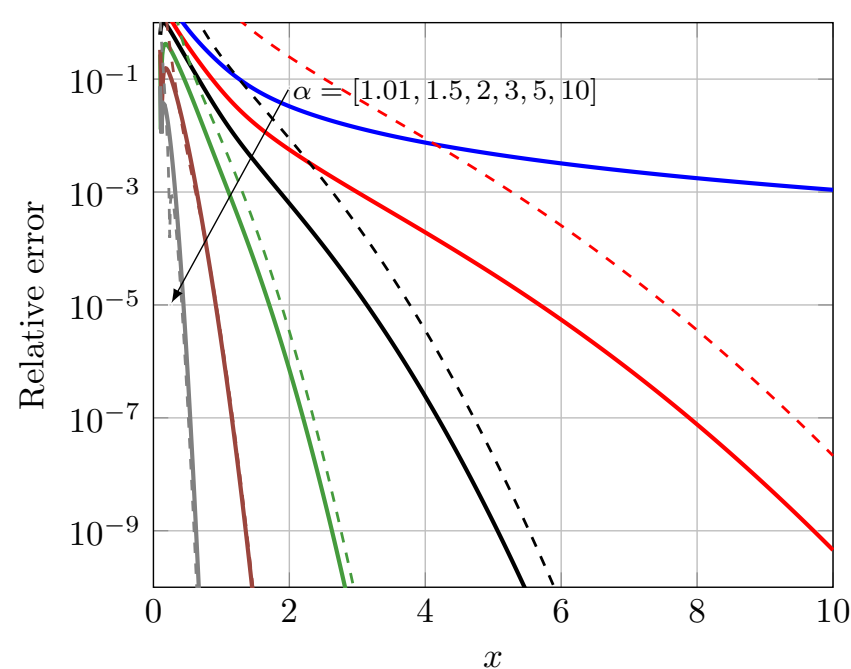

Fig. 1. Relative error between the exact evaluation of Marcum $Q$-function and the approximations in (3), in solid lines, and in (6), in dashed lines, for different values of the parameter $\alpha$ and $m=1$.

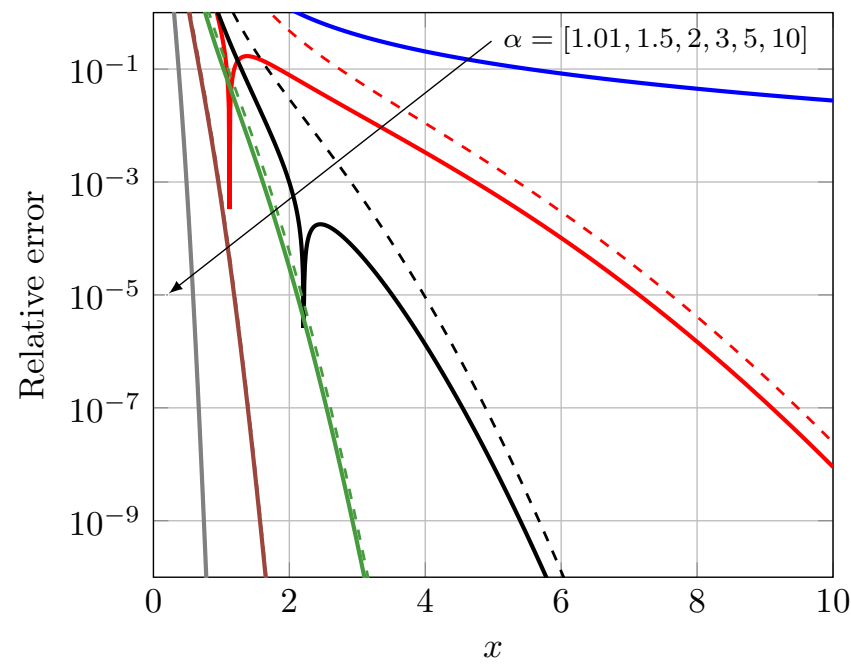

Fig. 2. Relative error between the exact evaluation of Marcum $Q$-function and the approximations in (3), in solid lines, and in (6), in dashed lines, for different values of the parameter $\alpha$ and $m=3$.

In Fig. 2, we evaluate $\varepsilon_{3}(\alpha, x)$ for different values of $\alpha$. A larger value of $m$ makes the approximation error to be larger for small values of $\alpha$. As $\alpha$ is increased, we observe how the approximation error is reduced. Hence, we can conclude that the approximation error slightly grows with $m$ for given values of $x$ and $\alpha$. Since the approximations (3) and (6) are asymptotically exact for large values of the arguments, the error decays as $\alpha \cdot x$ is increased.

\section{Applications}

In this Section, we use the approximations presented in this paper to gain additional insights into a number of scenarios in communication theory where the symmetric difference of Marcum $Q$-functions makes appearance.

\section{A. Statistical Characterization of Hoyt Fading}

The Hoyt distribution, which is indistinctly referred to as Nakagami- $q$ distribution in the literature [21], is often used to model the variation of the signal amplitude in different scenarios of wireless communications [14]. Basically, this model assumes that the in-phase and quadrature components are Gaussian random processes with zero-mean and arbitrary variances, and includes Rayleigh $(q=1)$ and one-sided Gaussian $(q=0)$ distributions as particular cases.

In [15], a closed-form expression was found for this cdf in terms of a symmetric difference of Marcum $Q_{1}$-functions as

$$
F_{X}(x)=Q_{1}\left(\alpha_{q} \frac{x}{\sqrt{\Omega}}, \beta_{q} \frac{x}{\sqrt{\Omega}}\right)-Q_{1}\left(\beta_{q} \frac{x}{\sqrt{\Omega}}, \alpha_{q} \frac{x}{\sqrt{\Omega}}\right),
$$

where $\Omega=\mathrm{E}\{X\}$ and $q \in[0,1]$ are known as the spread parameter and the shape parameter, respectively, and the parameters $\alpha_{q}$ and $\beta_{q}$ are in [22] given by

$$
\begin{aligned}
& \alpha_{q}=\frac{\sqrt{1-q^{4}}}{2 q} \sqrt{\frac{1+q}{1-q}}, \\
& \beta_{q}=\alpha_{q} \frac{1-q}{1+q} .
\end{aligned}
$$

Since the Hoyt distribution tends to the one-sided Gaussian distribution as $q \rightarrow 0$, one might intuitively think that the Hoyt cdf needs to be similar to the one-sided Gaussian cdf as $q$ is reduced. Using the approximation in (5) and after some algebra, we can approximate (8) in a very compact form as

$$
F_{X}(x) \approx 1-\frac{2}{\sqrt{1-q^{2}}} \mathrm{Q}\left(\frac{x}{\sqrt{\Omega}} \sqrt{1+q^{2}}\right) .
$$

And equivalently, we can express the Hoyt ccdf as

$$
\bar{F}_{X}(x) \approx \frac{2}{\sqrt{1-q^{2}}} \mathrm{Q}\left(\frac{x}{\sqrt{\Omega}} \sqrt{1+q^{2}}\right) .
$$

Interestingly, these expressions reduce to the exact onesided Gaussian distribution for $q=0$. In Fig. 3, the exact (lines) and approximated values (markers) for the Hoyt ccdf are represented as a function of $q$, for different values of the argument $x / \sqrt{(} \Omega)$. We can appreciate how (12) performs better as $q \rightarrow 0$ or $x / \sqrt{(} \Omega)$ grows, which is in concordance with the asymptotic approximations used in Appendix A.

\section{B. BER Analysis of Communication Systems}

One of the milestone papers in communication theory was published by Simon and Alouini [23], which served as a basis for the book "Digital Communications over Fading Channels" [14] that became one of the most referenced textbooks in the last decade. In this paper, whose contents were complemented in [13], the authors demonstrated that the error probability of different modulation schemes employing multichannel reception could be expressed in the following compact form

$$
P_{b}=\frac{1}{2}-\frac{1}{2^{2 N-1}} \sum_{n=1}^{N}\left(\begin{array}{c}
2 N-1 \\
N-n
\end{array}\right) \Delta \mathcal{Q}_{n}\left(a_{2} \sqrt{\gamma}, a_{1} \sqrt{\gamma}\right),
$$

where $a_{1}$ and $a_{2}$ are modulation dependent parameters, $\gamma$ denotes the total instantaneous SNR per bit and $N$ is the number of reception branches. For equal energy equiprobable 


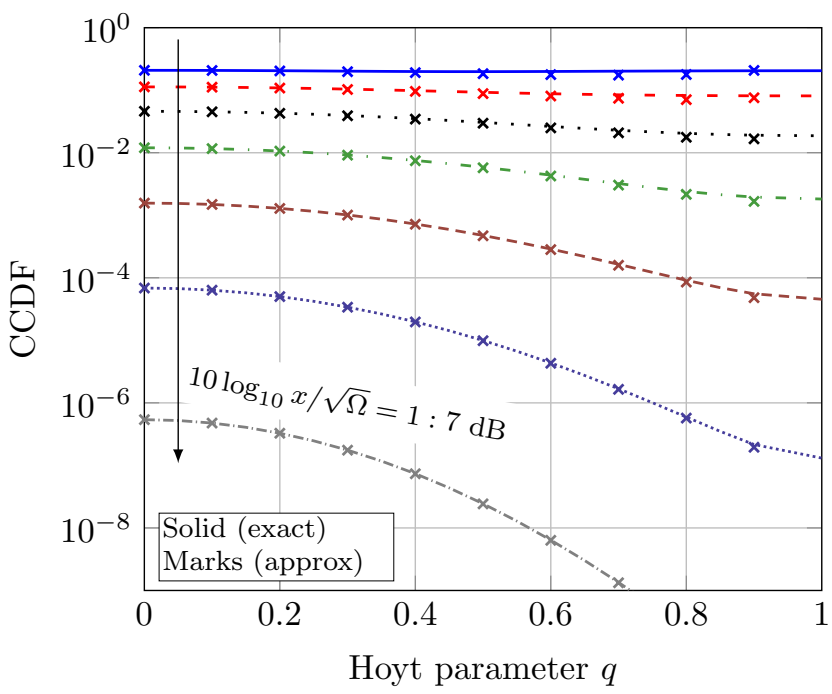

Fig. 3. Exact vs. approximated ccdf of the Hoyt distribution as a function of $q$, for different values of the argument. for different number of receiver branches $N$. Solid lines correspond are calculated using (8), whereas markers correspond to (11).

correlated binary signals with non-coherent reception, we have $a_{1}=\sqrt{\frac{1-\sqrt{1-|\rho|^{2}}}{2}}$ and $a_{2}=\sqrt{\frac{1+\sqrt{1-|\rho|^{2}}}{2}}$. In the case of QPSK modulation with Gray coding and differentially coherent detection, we have $a_{1}=\sqrt{2-\sqrt{2}}$ and $a_{2}=\sqrt{2+\sqrt{2}}$.

Since expression (13) is conveniently given in terms of a symmetric difference of generalized Marcum $Q$-functions, using (3) and after some algebra we can approximate the BER as

$$
P_{b}\left(N, \gamma, a_{1}, a_{2}\right) \approx C_{N}\left(a_{1}, a_{2}\right) \cdot Q\left(\left[a_{2}-a_{1}\right] \sqrt{\gamma}\right),
$$

where

$$
C_{N}\left(a_{1}, a_{2}\right) \triangleq \sum_{n=1}^{N}\left(\begin{array}{c}
2 N-1 \\
N-n
\end{array}\right)\left\{\left(\frac{a_{2}}{a_{1}}\right)^{n-\frac{1}{2}}+\left(\frac{a_{2}}{a_{1}}\right)^{\frac{1}{2}-n}\right\} \text {. }
$$

Interestingly, the coefficient $C$ is independent of $\gamma$. If we use the exponential approximation for the Gaussian $Q$-function, we obtain a new approximated expression for the BER as

$$
P_{b}\left(N, \gamma, a_{1}, a_{2}\right) \approx \frac{C_{N}\left(a_{1}, a_{2}\right)}{\sqrt{2 \pi}\left(a_{2}-a_{1}\right)} \frac{e^{-\frac{\left[a_{2}-a_{1}\right]^{2} \gamma}{2}}}{\sqrt{\gamma}} .
$$

In Fig. 4, the BER of multibranch QPSK receivers with differentially coherent detection is represented for different number of receive antennas $N$, as a function of the total instantaneous SNR per bit $\gamma$. We observe how even though approximation (15) outperforms approximation (16) for lowmedium values of $\gamma$, both are asymptotically exact as $\gamma$ is increased.

In Fig. 5, we represent the BER of correlated binary signals with non-coherent detection, for different values of the correlation parameter $\rho$ and $N=2$. As the correlation coefficient grows, the accuracy provided by approximation (15) is increased. As in the previous figure, we note how both approximations become asymptotically exact as $\gamma$ is increased.

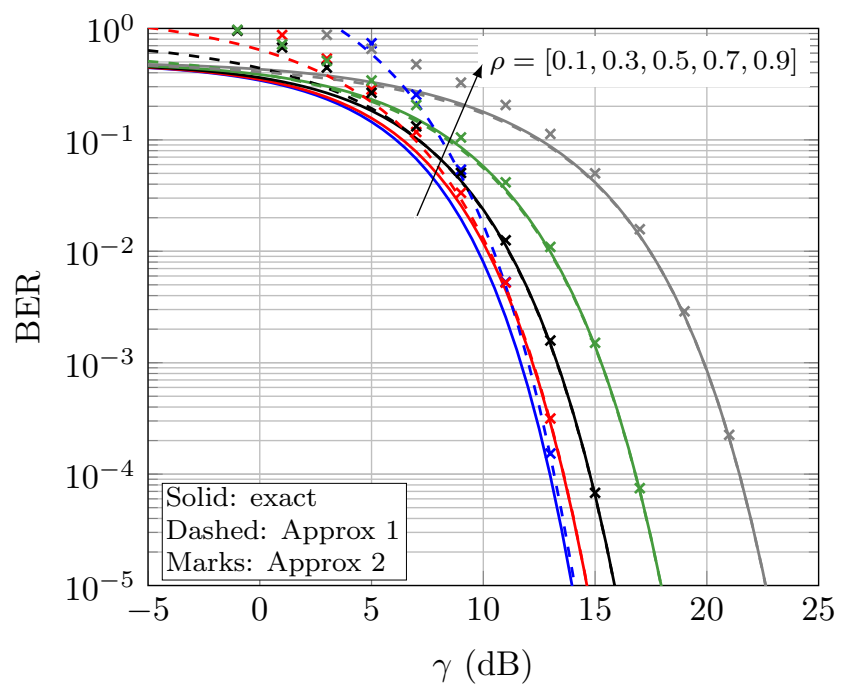

Fig. 4. Exact vs. approximated BER of multibranch DQPSK for different number of receiver branches $N$. Approximation 1 corresponds to eq. (3), and approximation 2 corresponds to eq. (6).

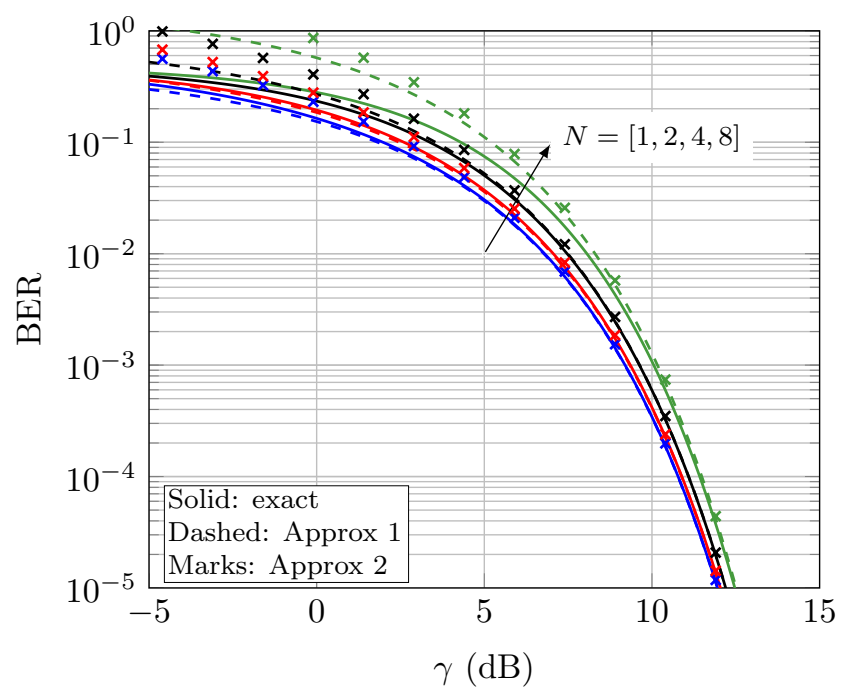

Fig. 5. Exact vs. approximated BER of non coherent binary detected correlated signals for different values of the correlation coefficient $\rho$ and $N=2$ receiver branches. Approximation 1 corresponds to eq. (3), and approximation 2 corresponds to eq. (6).

Hence, the exponential decay of the BER for the investigated communication systems where the error probability has the form of (13) has been stated.

\section{Level Crossing Statistics of a Sampled Rayleigh Envelope}

Second-order statistics of stationary random processes incorporate information related with the dynamic behavior of the process. In the context of fading channels, one example of such statistics is the level crossing rate (LCR), which characterizes how often the envelope fading passes a certain threshold.

The LCR of continuous Gaussian-like processes are usually characterized following Rice's approach [19], from the joint distribution of the envelope fading and its time derivative. If 


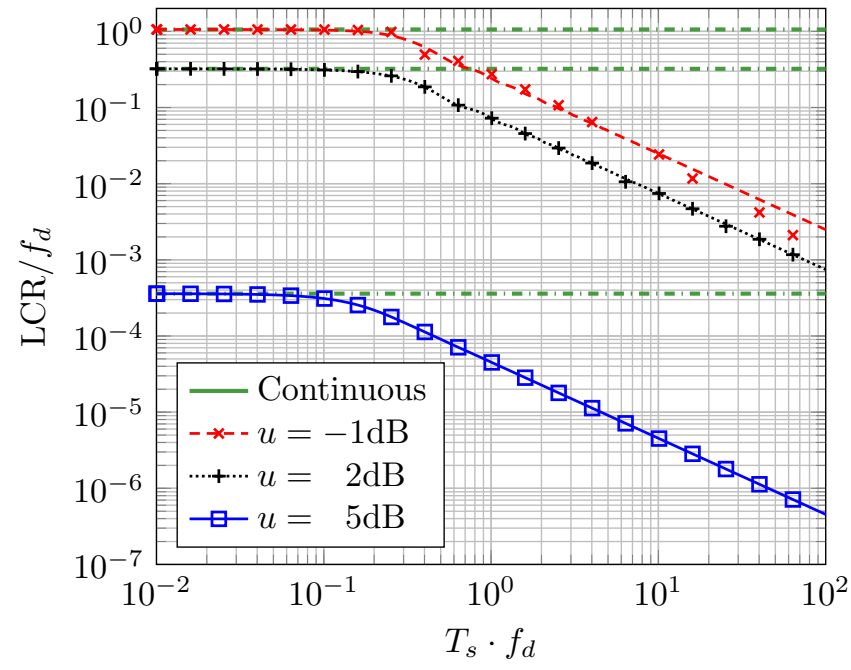

Fig. 6. LCR as a function of the normalized sampling period $T_{s} \cdot f_{D}$, for different values of the threshold value $u$.

the random process of interest is sampled at a finite period $T_{s}$, the probability of missing a level crossing between two samples is non-null; hence, the LCR of a sampled random process should necessarily be lower than the LCR of the continuous version of the process. This aspect was recently addressed in [18], where the LCR of a sampled random process was expressed in terms of the cdf and the bivariate cdf of the envelope fading $Z$ as

$$
N_{Z}\left(u, T_{s}\right)=\frac{F_{Z}(u)-F_{Z_{1}, Z_{2}}\left(u, u ; \rho_{T_{s}}\right)}{T_{s}},
$$

where $u$ is the threshold level, $Z_{1}=Z(t), Z_{2}=Z\left(t+T_{s}\right)$ and $\rho_{T_{s}}$ is the correlation coefficient between $Z_{1}$ and $Z_{2}$. In the following, we will omit the subindex $T_{s}$ in $\rho$ for notational simplicity. Using the results in [18], the exact LCR of a sampled Rayleigh fading envelope is given by

$$
N_{Z}(u)=\frac{e^{-u^{2}}}{T_{s}}\left(Q_{1}\left(k_{\rho} u, k_{\rho} u|\rho|\right)-Q_{1}\left(k_{\rho} u|\rho|, k_{\rho} u\right)\right),
$$

where $k_{\rho}=\sqrt{\frac{2}{1-|\rho|^{2}}}$. Since $|\rho|<1$, the symmetric difference of Marcum $Q$-functions in (18) can be approximated using (3). Hence, the LCR in this scenario can be approximated as

$N_{Z}(u)=\frac{e^{-u^{2}}}{T_{s}}\left(1-\left[\sqrt{|\rho|}+\sqrt{|\rho|^{-1}}\right] Q\left(u \sqrt{2 \frac{1-|\rho|}{1+|\rho|}}\right)\right)$

In Fig. 6, the LCR of a sampled Rayleigh envelope is represented as a function of the sampling period $T_{s}$. A correlation profile following Clarke's model is considered, and both axes are normalized by the Doppler frequency $f_{D}$. The dotteddashed green line corresponds to the LCR of a continuous Rayleigh envelope [19], which is clearly independent of $T_{s}$. The rest of lines indicate the exact LCR of a sampled Rayleigh envelope given by (17), whereas the markers correspond to the approximated LCR given by (18). We observe how the approximation is extremely accurate for large values of the threshold level $u$ even for $T_{s} \cdot f_{D}>>1$, which corresponds to low correlated fading samples. For lower values of $u$, the approximation is still reasonably good for most values of $T_{s} \cdot f_{D}$. In agreement with the definition of the LCR of a sampled random process, we note how both the exact and approximated expressions tend to the LCR of the continuous random process as $T_{s} \cdot f_{D} \rightarrow 0$.

\section{Asymptotic Approximation of the Rice $I_{e}$ function}

The Rice $I_{e}$ function, defined as

$$
I_{e}(k, x) \triangleq \int_{0}^{x} e^{-t} I_{0}(k t) d t,
$$

has been largely used in many scenarios of interest in communication theory such as zero crossing problems [19] or in the analysis of angle modulation systems [24].

In [16], Pawula established a connection between the Rice $I_{e}$ function and the Marcum $Q$-function. Although not explicitly given in [16], both functions are related through the compact expression [25]

$$
I_{e}(k, x)=\frac{1}{\sqrt{1-k^{2}}} \Delta \mathcal{Q}_{1}(a, b),
$$

where $a=\sqrt{x\left(1+\sqrt{1-k^{2}}\right)}$ and $b=\sqrt{x\left(1-\sqrt{1-k^{2}}\right)}$. Hence, two direct approximations for $I_{e}$ can be obtained using (3) and (6) as

$$
\begin{aligned}
& I_{e}(k, x) \approx \frac{1}{\sqrt{1-k^{2}}}\left[1-\delta_{k} Q(a-b)\right], \\
& I_{e}(k, x) \approx \frac{1}{\sqrt{1-k^{2}}}\left[1-\frac{\delta_{k}}{\sqrt{2 \pi}(a-b)} e^{-\frac{(a-b)^{2}}{2}}\right],
\end{aligned}
$$

where

$$
\delta_{k}=\sqrt{\frac{1+\sqrt{1-k^{2}}}{1-\sqrt{1-k^{2}}}}+\sqrt{\frac{1-\sqrt{1-k^{2}}}{1+\sqrt{1-k^{2}}}} .
$$

It is worth mentioning that the author in [16] derived an approximated expression for an integral function closely related to $I_{e}$ in terms of the complementary error function. Even though his approach and the one followed in eq. (22) are completely different, both final approximations are coincident.

In Fig. 7, we study the approximation error of (22) and (23) using the definition in (7), for different values of the parameters $k$ and $x$. In general, the error tends to decay as $x$ is increased. We note how even though approximation (22) provides a better accuracy for a wider range of values of $k$, the approximation in (23) has a better behavior for some set of parameters where $k$ takes low values. However, the accuracy of (23) is clearly degraded as $k \rightarrow 1$.

\section{CONCLUSION}

The generalized Marcum $Q$-function appears in many scenarios in communication theory in the very special form of a symmetric difference. We have provided two simple approximations for this combination of generalized Marcum $Q$ functions, which are asymptotically exact and easy to evaluate. These results have been useful to analyze different problems which admit a solution in terms of the symmetric difference of generalized Marcum $Q$-functions. 


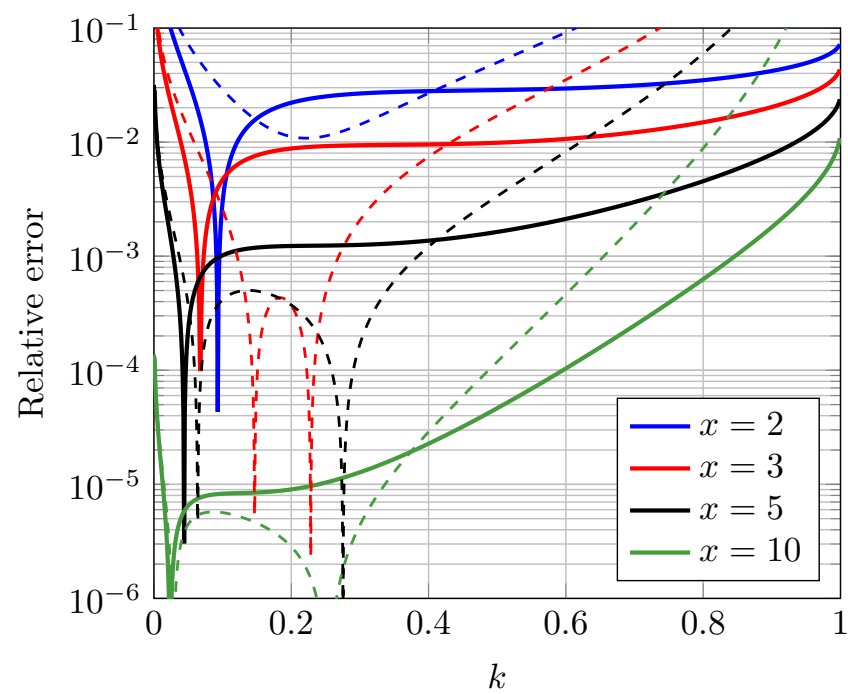

Fig. 7. Approximation error for the Rice $I_{e}(k, x)$ function using the approximations corresponding to eq. (22), in solid lines, and to (23), in dashed lines, for different values of $k$ and $x$.

\section{APPENDIX. PROOF OF LEMMA 1.}

Let us consider the symmetric difference of Marcum $Q$ functions defined in (2). Using the following equivalence given in $[26$, eq. 6]

$$
Q_{m}(a, b)+Q_{m}(b, a)=1+e^{-\frac{a^{2}+b^{2}}{2}} \sum_{i=1-m}^{m-1}\left(\frac{a}{b}\right)^{i} I_{i}(a b),
$$

we can express

$$
\Delta \mathcal{Q}_{m}(a, b)=1-2 Q_{m}(b, a)+e^{-\frac{a^{2}+b^{2}}{2}} \sum_{i=1-m}^{m-1}\left(\frac{a}{b}\right)^{i} I_{i}(a b) .
$$

Widely used in communication theory, the asymptotic relationship between the generalized Marcum $Q$-function and the Gaussian $Q$-function is given in $[14$, p. 100],

$$
Q_{m}(b, a) \approx\left(\frac{a}{b}\right)^{m-0.5} Q(a-b), \quad a>b, a \rightarrow \infty .
$$

This expression can be regarded as the first term of the asymptotic expansion in [27, eq. 3.6]. Using the first term of the Hankel expansion of $e^{-z} I_{m}(z)=\frac{1}{\sqrt{2 \pi z}}+\mathcal{O}\left(z^{-3 / 2}\right), z \rightarrow \infty$ in [28, eq. 10.40.1], and the first term of the asymptotic expansion of the complementary error function [20, eq. 7.1.23] $\operatorname{erfc}(z)=\frac{e^{-z^{2}}}{\sqrt{\pi} z}+\mathcal{O}\left(z^{-3} e^{-z^{2}}\right), z \rightarrow \infty$, we have an asymptotic relationship for the modified Bessel function of the first kind and the Gaussian $Q$-function

$$
e^{-\frac{\left(a^{2}+b^{2}\right)}{2}} I_{m}(a b) \approx \frac{a-b}{\sqrt{a b}} Q(a-b), \quad a \cdot b \rightarrow \infty .
$$

Both (27) and (28) are asymptotically exact for large values of their arguments, and yield

$$
\Delta \mathcal{Q}_{m}(a, b) \approx 1-\left\{2\left(\frac{a}{b}\right)^{m-0.5}-\frac{a-b}{\sqrt{a b}} \sum_{i=1-m}^{m-1}\left(\frac{a}{b}\right)^{i}\right\} Q(a-b),
$$

where we also assumed that the arguments $a$ and $b$ are sufficiently large, but their ratio $a / b$ remains finite. Solving the finite geometric series in (29) and after some algebra, we obtain

$$
\Delta \mathcal{Q}_{m}(a, b) \approx 1-[\overbrace{\left(\frac{a}{b}\right)^{m-0.5}+\left(\frac{a}{b}\right)^{0.5-m}}^{\kappa_{m}(a, b)}] Q(a-b) .
$$

We must note that an alternative derivation can be obtained if we extend the definition of the generalized Marcum $Q$ function to negative values of the order $m$ as in [29]: using the relation given by $\Delta \mathcal{Q}_{m}(a, b)=1-Q_{1-m}(b, a)$, we can express $\Delta \mathcal{Q}_{m}(a, b)=1-Q_{1-m}(b, a)-Q_{m}(b, a)$, and directly plugging the asymptotic relation between the generalized Marcum $Q$-function in (27) also yields the desired expression.

\section{REFERENCES}

[1] J. Marcum, Table of $Q$ Functions. Memorandum (Rand Corporation), Rand Corporation, 1950.

[2] C. Helstrom, Statistical Theory of Signal Detection. Macmillan, 1960.

[3] J. Marcum, "A statistical theory of target detection by pulsed radar," IRE Trans. Inf. Theory, vol. 6, no. 2, pp. 59-267, 1960.

[4] M. Schwartz, W. R. Bennett, and S. Stein, Communication systems and techniques. McGraw-Hill, New York :, 1966.

[5] F. J. Lopez-Martinez, D. Morales-Jimenez, E. Martos-Naya, and J. F. Paris, "On the Bivariate Nakagami-m Cumulative Distribution Function: Closed-Form Expression and Applications," IEEE Trans. Commun., vol. 61, no. 4, pp. 1404-1414, 2013.

[6] D. Morales-Jimenez, F. J. Lopez-Martinez, E. Martos-Naya, J. F. Paris, and A. Lozano, "Connections between the Generalized Marcum QFunction and a class of Hypergeometric Functions," IEEE Trans, Inf. Theory, vol. 60, pp. 1077-1082, Feb 2014.

[7] J. Proakis, "On the Probability of Error for Multichannel Reception of Binary Signals," IEEE Trans. Comm. Technol., vol. 16, no. 1, pp. 68-71, 1968.

[8] M. K. Simon and M.-S. Alouini, "Exponential-type bounds on the generalized Marcum Q-function with application to error probability analysis over fading channels," IEEE Trans. Commun., vol. 48, no. 3, pp. 359-366, 2000.

[9] G. Corazza and G. Ferrari, "New bounds for the Marcum $Q$-function," IEEE Trans. Inf. Theory, vol. 48, pp. 3003-3008, Nov. 2002.

[10] A. Baricz and Y. Sun, "New Bounds for the Generalized Marcum $Q$ Function," IEEE Trans. Inf. Theory, vol. 55, pp. 3091-3100, July 2009.

[11] V. M. Kapinas, S. K. Mihos, and G. K. Karagiannidis, "On the Monotonicity of the Generalized Marcum and Nuttall Q -Functions," IEEE Trans. Inf. Theory, vol. 55, pp. 3701 -3710, Aug. 2009.

[12] Y. Sun;, A. Baricz;, and S. Zhou, "On the Monotonicity, Log-Concavity, and Tight Bounds of the Generalized Marcum and Nuttall $Q$-Functions," IEEE Trans. Inf. Theory, vol. 56, pp. 1166-1186, Mar. 2010.

[13] M. K. Simon and M.-S. Alouini, "A unified approach to the probability of error for noncoherent and differentially coherent modulations over generalized fading channels," IEEE Trans. Commun., vol. 46, no. 12, pp. 1625-1638, 1998.

[14] M. K. Simon and M.-S. Alouini, Digital Communication over Fading Channels (Wiley Series in Telecommunications and Signal Processing). Wiley-IEEE Press, 2005.

[15] J. F. Paris, "Nakagami-q (Hoyt) distribution function with applications," Electronics Letters, vol. 45, no. 4, pp. 210-211, 2009.

[16] R. F. Pawula, "Relations between the Rice Ie-function and the Marcum Q-function with applications to error rate calculations," Electronics Letters, vol. 31, no. 24, pp. 2078-2080, 1995.

[17] M. E. Hellman, R. A. Flower, B. K. Levitt, and R. S. Kennedy, "Processing and Transmission of Information," Quarterly Progress Report 99, Research Laboratory of Electronics, Massachusetts Institute of Technology, Cambridge, CT, Oct 1970.

[18] F. J. Lopez-Martinez, E. Martos-Naya, J. F. Paris, and U. FernandezPlazaola, "Higher order statistics of sampled fading channels with 
applications," IEEE Trans. Veh. Technol., vol. 61, no. 7, pp. 3342-3346, 2012.

[19] S. O. Rice, Mathematical analysis of random noise. Bell Telephone Laboratories, 1944.

[20] M. Abramowitz and I. Stegun, Handbook of Mathematical Functions. Dover Publications, 1965.

[21] R. S. Hoyt, "Probability Functions for the Modulus and Angle of the Normal Complex Variate," Bell System Technical Journal, vol. 26, no. 2, pp. 318-359, 1947.

[22] J. F. Paris, "Erratum to Nakagami-q (Hoyt) distribution function with applications," Electronics Letters, vol. 45, no. 8, p. 432, 2009.

[23] M. K. Simon and M.-S. Alouini, "A unified approach to the performance analysis of digital communication over generalized fading channels," Proceedings of the IEEE, vol. 86, no. 9, pp. 1860-1877, 1998.

[24] R. F. Pawula, S. O. Rice, and J. Roberts, "Distribution of the phase angle between two vectors perturbed by Gaussian noise," IEEE Trans. Commun., vol. 30, pp. 1828 - 1841, Aug 1982.
[25] P. Sofotasios and S. Freear, "Upper and lower bounds for the Rice Iefunction," in Australasian Telecommunication Networks and Applications Conference (ATNAC), 2011, pp. 1-4, 2011.

[26] A. H. Nuttall, "Some Integrals Involving the (Q sub M)-Function," Tech. Rep. 779 846, Naval Underwater Systems Center, New London, CT, May 1974.

[27] N. Temme, "Asymptotic and numerical aspects of the noncentral chi-square distribution," Computers \& Mathematics with Applications, vol. 25, no. 5, pp. 55-63, 1993.

[28] F. W. J. Olver, D. W. Lozier, R. F. Boisvert, and C. W. Clark, NIST Handbook of Mathematical Functions. New York, NY: Cambridge University Press, 2010.

[29] C. O'Driscoll and C. Murphy, "A simplified expression for the probability of error for binary multichannel communications," IEEE Trans. Commun., vol. 57, pp. 32-35, Jan. 2009. 